\title{
Pandemic Response to COVID-19: Lessons from Restructuring Breast Imaging at a Multi-site Academic Center in New York City
}

\author{
Ami D. Shah ${ }^{1}$ (1) $\cdot$ Katharine D. Maglione ${ }^{1} \cdot$ Lisa Abramson $^{1} \cdot$ Laurie R. Margolies $^{1}$
}

Accepted: 19 August 2021 / Published online: 29 September 2021

(C) The Author(s), under exclusive licence to Springer Science+Business Media, LLC, part of Springer Nature 2021

\begin{abstract}
Purpose of Review The COVID-19 pandemic has been an unprecedented challenge for healthcare delivery, disrupting normal workflows and demanding re-allocation of resources. In the setting of breast imaging, this entailed an initial marked reduction of services during the acute phase of the pandemic followed by a gradual reopening with modified protocols to minimize potential virus exposure.

Recent Findings Protocols were devised for providing limited breast imaging services during the acute phase of the pandemic and for safely reopening during the post acute phase. Example cases of breast cancers diagnosed during the acute phase of the pandemic are provided to illustrate the modified workflow.

Summary This manuscript reviews practical strategies for restructuring breast imaging workflow, onsite policies, and staffing at a multi-site academic center in New York City during the COVID-19 pandemic. By sharing our experience, we hope to provide others with a roadmap for managing breast imaging services during future unforseen crises.
\end{abstract}

Keywords COVID-19 · Pandemic · Breast imaging · Workflow

This article is part of the Topical collection on Breast Imaging.

Ami D. Shah

ami.shah@mountsinai.org

1 Department of Diagnostic, Molecular, and Interventional Radiology, Icahn School of Medicine at Mount Sinai, New York, NY, USA

\section{Introduction}

On March 1, 2020, the first COVID-19 case in New York State was identified, linked to travel to Iran, a country known to have widespread contemporaneous coronavirus transmission [1]. On March 3, 2020, the first confirmed non-travel-related COVID-19 case was identified in NY State in an individual who lived in Westchester County and worked in New York City [2]. This was followed by rapid exponential growth of confirmed cases in New York State, resulting in the declaration of a State of Emergency by Governor Andrew Cuomo on March 7, 2020 and a statewide stay at home order on March 20, 2020 [3, 4]. The stay at home order mandated the closure of all non-essential businesses and all non-essential gatherings. The closure of non-essential businesses included elective care and nonurgent medical procedures, including breast cancer screening services.

It was against this backdrop that our department urgently re-structured our multi-site academic breast imaging services. At the time, there were no published guidelines for managing breast health services during a pandemic. As such, our physicians, administrators, and staff collaborated closely to rapidly devise and deploy appropriate policies and procedures for delivering care during this unprecedented crisis.

This manuscript shares strategies used at our multi-site academic facility in New York City for restructuring breast imaging workflow, onsite protocols, and staffing to allow for optimal patient care while minimizing risk during the acute phase of the COVID-19 pandemic and for safe reopening during the post-acute peri-pandemic period. By sharing our experience, we hope to offer others a roadmap for managing breast imaging services during future unforeseen crises. 


\section{Acute Phase of the Pandemic}

During the acute phase, defined as the period during which New York State experienced a rapid exponential growth in COVID-19 cases, our protocols were modified to minimize potential virus exposure and conserve resources, including personal protective equipment, staff, and limited COVID19 testing capacity. This can be thought of as the "blunt instrument" phase of our response, where simple, straight forward, and quickly deployable protocols were devised and implemented to achieve our goals.

\section{Workflow}

Cases were triaged into two groups: those requiring immediate attention and those which should be postponed. All routine screening breast imaging exams were postponed. Routine diagnostic cases, defined as imaging performed for post-treatment surveillance, 6 months followup imaging, and evaluation of non-focal breast pain, were postponed. Scheduled breast biopsies were reviewed by attending radiologists, and those designated as low suspicion for malignancy (BI-RADS 4a, < 10\% likelihood of malignancy) were deferred. Scheduling and front desk staff contacted these patients to reschedule their exams for 3 months in the future, with a plan to re-assess and implement further delays, if needed.

Urgent diagnostic cases were expedited and included patients with newly diagnosed breast cancer, suspected abscess, newly palpable masses, suspicious nipple discharge, and BI-RADS 0 screening exam callbacks. Patients with pending biopsies for lesions deemed BI-RADS $4 \mathrm{~b}$, $4 \mathrm{c}$, and 5 (10\% or greater suspicion for malignancy) were also expedited. Cases deemed appropriate for expedited breast imaging exams and procedures were pre-screened for COVID-19 symptoms by phone prior to their appointments and onsite at the facility entrance. Patients experiencing symptoms had their exams delayed by at least 2

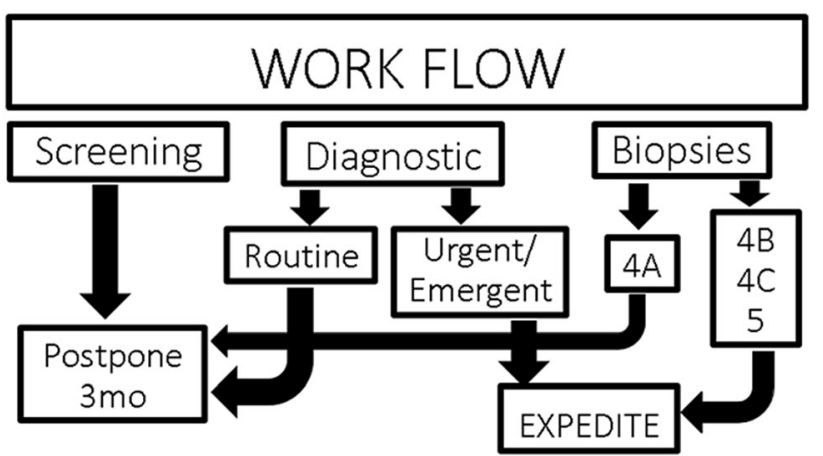

Fig. 1 Diagram of modified workflow during acute phase of the pandemic weeks, given the known incubation period of the virus. Figure 1 summarizes our modified workflow.

\section{Onsite Protective Measures}

New rules regarding the use of personal protective equipment were implemented to minimize potential virus transmission onsite, including mandatory surgical masks for all staff and patients. Social distancing was enforced through new signage posted throughout the facility and gentle verbal reminders to both staff and patients. Social distancing was also facilitated by decreased density of personnel and patients onsite resulting from changes in workflow and staffing protocols. Figure 2 illustrates this decrease in density. In-person exchanges were reduced by communicating by phone whenever possible, such as when checking cases and relaying biopsy recommendations to patients.

\section{Staffing}

With the changed workflow and resulting marked decrease in volume, an oversupply of staff had to be managed. Some breast imaging attendings, due to health concerns and childcare issues, took voluntary unpaid leaves. The remaining attendings were encouraged to use vacation days and conference time. Academic time was also given to develop abstracts, papers, and resident teaching files. When two or more attendings were assigned to a single site, they were permitted to split their shifts so that only one radiologist was in the reading room at a time. Attending site assignments were made based on proximity to home to reduce potential exposure while commuting such that most were able to walk or bike to work.

Residents assigned to breast imaging were re-assigned to either emergency department or inpatient radiology rotations. Others were re-assigned to inpatient medical teams. All resident lectures were transitioned to online platforms.

Technologist shifts were staggered. Some technologists and support staff took leave due to childcare needs. Technologists were encouraged to make use of space in empty exam rooms to reduce breakroom density.

\section{Acute Phase Protocols in Practice: Case Examples}

\section{Case 1: Expedited Workflow}

New architectural distortion was identified in the upper outer left breast on a screening mammogram in a woman in her 50 s with a positive family history of breast cancer in her mother in her 50s (Fig. 3a). While most screening 
Fig. 2 Illustration of decreased onsite density resulting from workflow and staffing changes

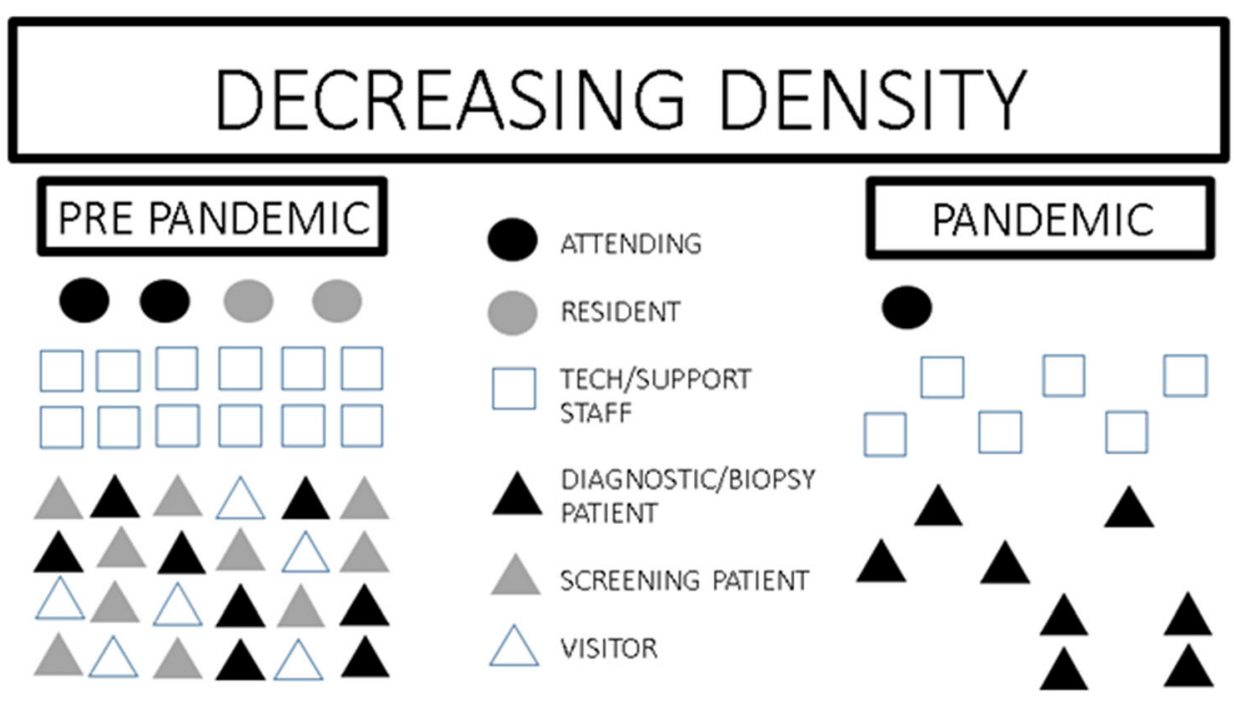

exams were postponed, if a patient was reluctant to delay her exam, particularly if high risk, we would make accommodations. This patient presented early in the course of the pandemic, as we were transitioning to the new workflow protocols. The patient had left the department before her screening mammogram was reviewed by the radiologist, as the technologist who performed the exam was following our routine pre-pandemic workflow for screening exams. She was immediately called back, and diagnostic imaging was performed the next day. This demonstrated a highly suspicious $6 \times 9 \times 6 \mathrm{~mm}$ shadowing, irregular, non-parallel mass on ultrasound corresponding to the new mammographic distortion (Fig. 3b). Same-day ultrasound-guided biopsy was performed and yielded invasive ductal carcinoma. Pathology results were available within $24 \mathrm{~h}$ of biopsy. $24 \mathrm{~h}$ after the biopsy ( $48 \mathrm{~h}$ after the initial screening exam), the patient underwent breast MRI to evaluate the extent of disease. The MRI demonstrated no multifocal, multicentric, or contralateral disease (Fig. 3c). Wire localization and lumpectomy was performed 4 days later. Detection, workup, diagnosis, and treatment of this patient's breast cancer were performed within 7 days. As a result of this case, we amended our workflow policies to address the few screening exams which were performed, such as this high-risk patient who was reluctant to delay her exam. Technologists were instructed to present these cases for reads before the patient left the department, so that any necessary workups could be performed the same day.

\section{Case 2: Delayed Workflow}

A woman in her 60s underwent screening mammography through our mobile van mammography program the week before the state-wide stay at home order and closure of breast screening services. Review of her mammogram demonstrated a highly suspicious mass in her upper outer right breast (Fig. 4a). An appointment for diagnostic imaging and same-day biopsy was scheduled for the patient the following week, after the new workflow was implemented. During a phone call to pre-screen the patient the day before her appointment, she reported symptoms suspicious for possible COVID-19. Per the new workflow protocol, her exam was postponed 2 weeks. Her diagnostic imaging was further delayed, as a family member contracted COVID-19 and was unable to bring the patient in for her appointment. Arrangements were subsequently made through our mobile mammography program to provide the patient with transportation to and from the imaging center for her diagnostic imaging and biopsy. Three months after the initial screening exam, diagnostic imaging demonstrated a highly suspicious irregular hypoechoic nonparallel mass with indistinct margins in the 10 o'clock right breast and an abnormal right axillary lymph node, both of which underwent ultrasound-guided biopsies the same day(Fig. 4b). Pathology demonstrated invasive ductal carcinoma metastatic to the right axilla.

\section{Case 3: Urgent Exam}

A woman in her early 20 s presented for evaluation of a palpable area of concern in the 6:00 left breast and a 3 months history of bloody left nipple discharge. The patient was initially evaluated with bilateral whole breast ultrasound which was read remotely due to reduced staffing. No abnormality was detected. A recommendation was made for breast surgical consultation for further evaluation of the patient's suspicious clinical symptoms. The patient presented for breast surgical consultation the following day at our breast cancer center. A same-day diagnostic 


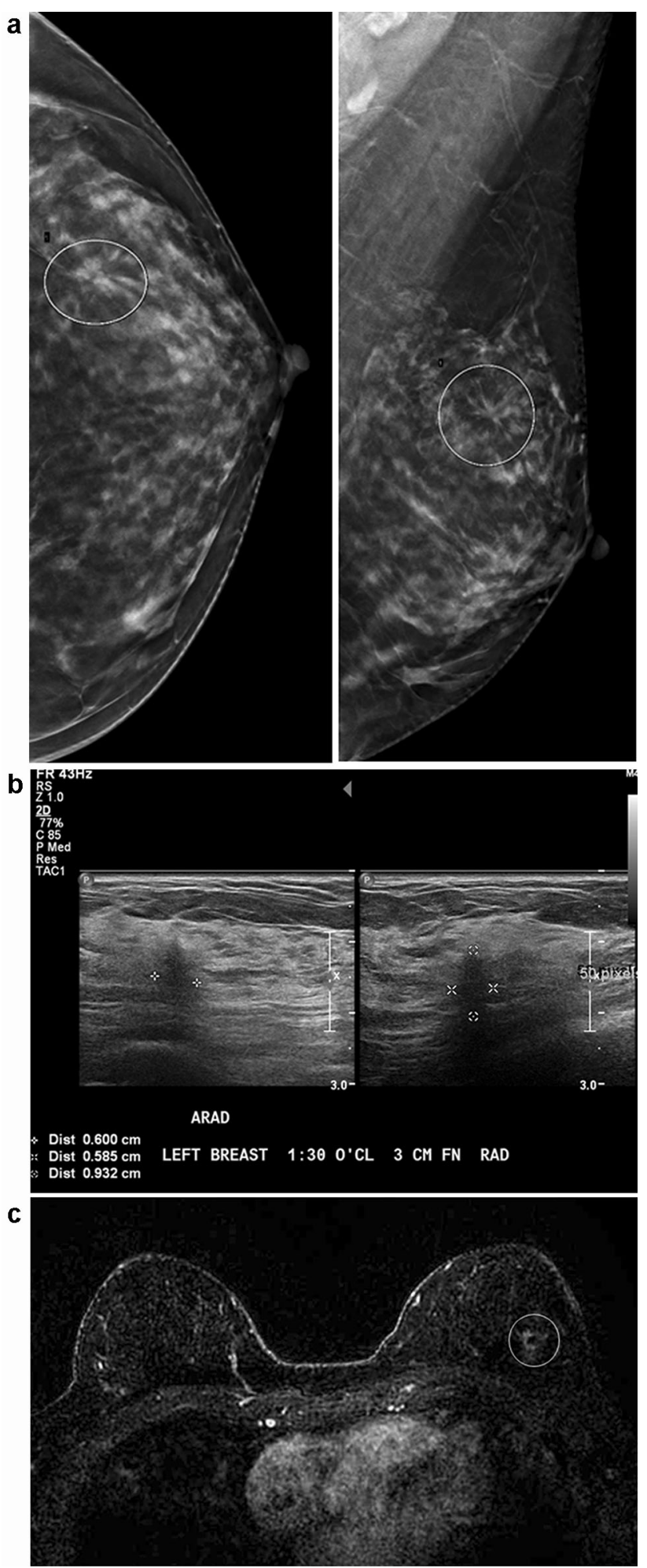

4Fig. 3 a Screening mammogram detected left breast architectural distortion in a woman in her 50s. b Ultrasound demonstrates a highly suspicious $6 \times 9 \times 6 \mathrm{~mm}$ shadowing non parallel mass with indistinct margins correlating with mammographic distortion. Same-day ultrasound-guided biopsy yielded invasive ductal carcinoma. c Expedited breast MRI for extent of disease assessment demonstrates no multifocal, multicentric, or contralateral disease. Circle indicates known biopsy-proven carcinoma with biopsy clip artifact

mammogram was performed, demonstrating highly suspicious segmental amorphous and pleomorphic calcifications spanning $4.2 \mathrm{~cm}$ at the site of palpable concern (Fig. 5). Stereotactic biopsy was performed the next morning; pathology results were available $24 \mathrm{~h}$ later and demonstrated ductal carcinoma in situ. Time from initial presentation to diagnosis in this patient was $72 \mathrm{~h}$.

\section{Re-opening and Operating in the Post-acute Phase}

In early May 2020, Governor Cuomo announced plans for a four-phase Statewide reopening, establishing regional metrics for infection rate, hospital capacity, testing capacity, and contact tracing to permit entry into each phase [5]. On June 8, 2020, New York City met criteria to enter the first phase of reopening, which permitted resumption of elective surgery and ambulatory care services [6]. As compared to the "blunt instruments" used for closure of breast imaging services at our multi-site academic facility in New York City, reopening involved fine, carefully calibrated incremental changes.

We began our reopening by expediting diagnostic and screening patients whose appointments were postponed. Fortunately, most had already been given appointments starting in mid-June 2020 when appointments were initially rescheduled at the start of the pandemic in midMarch 2020. Our first and foremost priority was to ensure the safety of staff and patients as we reopened. New templates were designed to accommodate social distancing, visitors were not permitted, capacity limits were established for waiting rooms, and mask compliance was enforced. Initially, while volume remained low, screening exams were checked before patients left the department, with any required diagnostic workups performed the same day. As volume increased, we reverted back to our "screen and go" policy, as those patients waiting for screening exams to be read resulted in an untenable increase in density in our waiting areas.

Patients were initially hesitant to resume in-person care, particularly for screening exams and routine diagnostic follow-up exams. These patients were reassured through direct phone calls, as well as television and social media advertising campaigns. By September 2020, monthly patient volume had increased to $105 \%$ as compared to 
Fig. 4 a Screen detected highly suspicious mass detected in the upper outer right breast through mobile van mammography program in a woman in her $60 \mathrm{~s}$ just before pandemic-related workflow changes were implemented. b Diagnostic imaging, delayed secondary to concerns regarding COVID-19 symptoms and transportation issues, performed 3 months after initial screening exam demonstrates a highly suspicious 2.7 mass with indistinct margins at 10:00 $12 \mathrm{~cm}$ from the nipple corresponding to the screendetected mammographic mass and an abnormal right axillary lymph node. Subsequent biopsy yielded invasive ductal carcinoma metastatic to the right axilla a
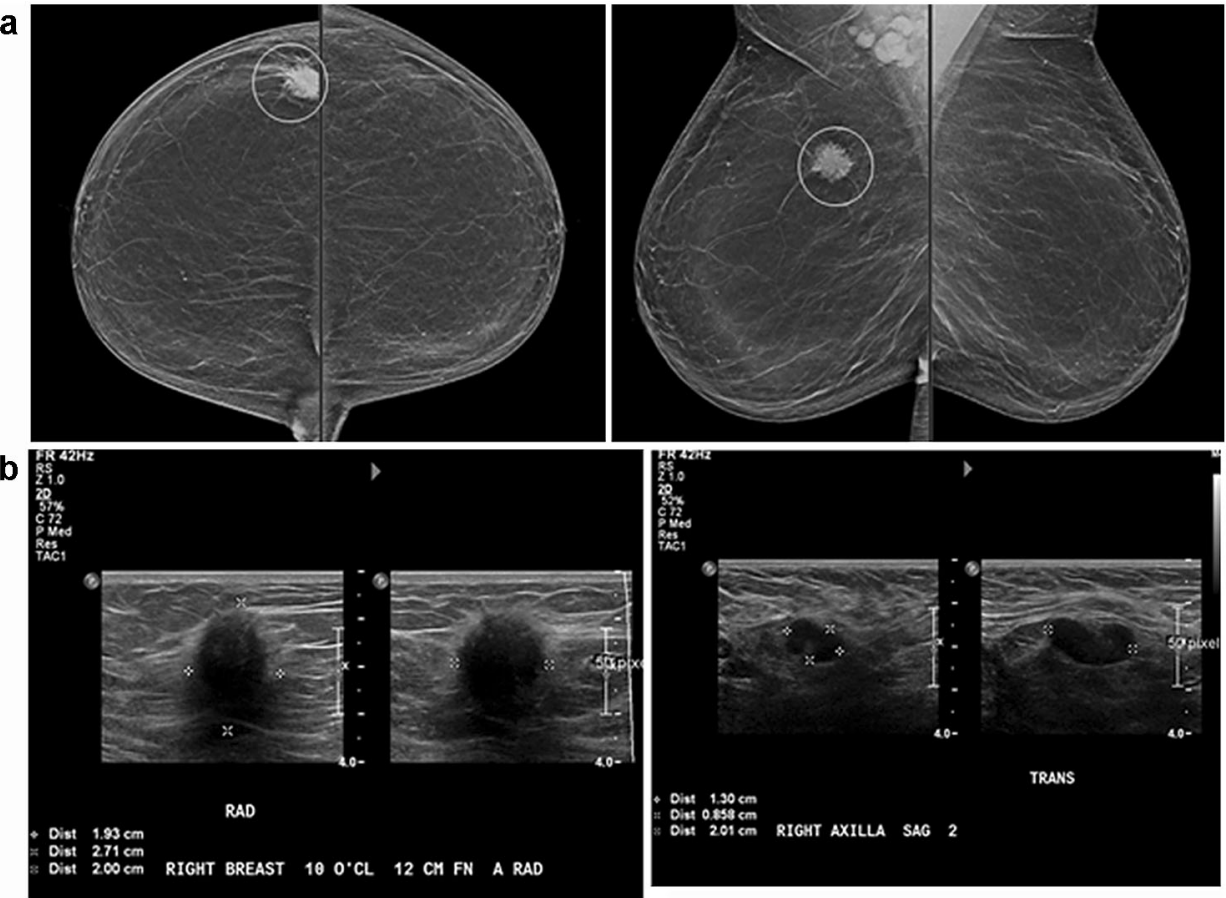
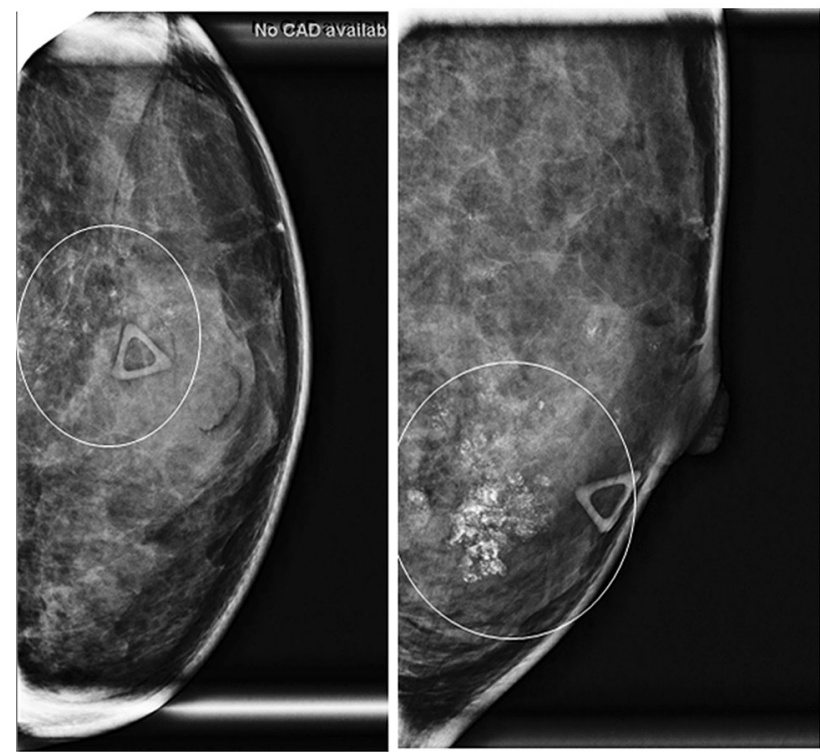

Fig. 5 A woman in her early 20s presents with a palpable area of concern in the 6:00 left breast and bloody nipple discharge $\times 3$ months. Diagnostic mammogram demonstrates highly suspicious segmental amorphous and pleomorphic calcifications spanning $4.2 \mathrm{~cm}$. Stereotactic biopsy yielded ductal carcinoma in situ

2019 (Fig. 6). In order to accommodate these patients while still allowing for adequate social distancing, evening hours and weekend hours were expanded.

With the advent of COVID-19 vaccinations in late December 2020, COVID-19 vaccine-related axillary adenopathy became an additional factor to consider in our

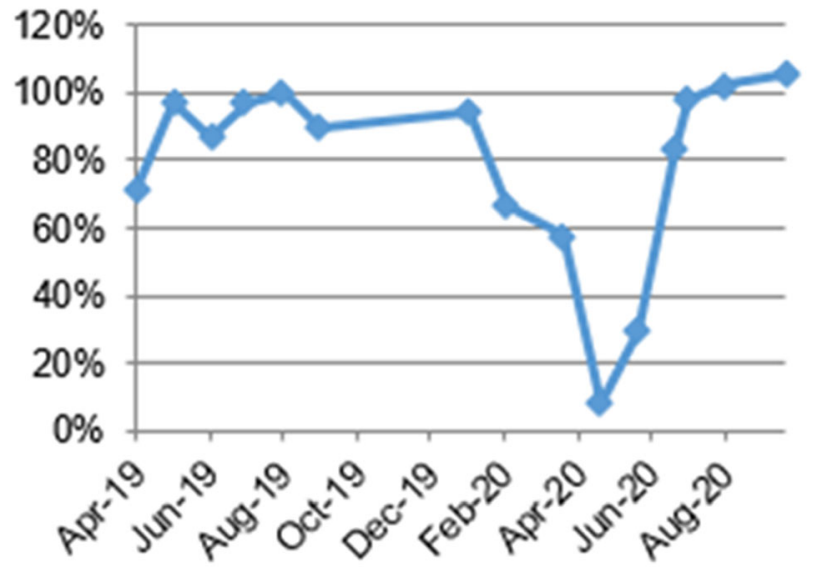

Fig. 6 Breast imaging monthly volume plotted as a percentage of the monthly volume from 1 year prior. After an acute drop in volume during the peak of the pandemic, volume of breast imaging cases rapidly rebounded, such that by September 2020, volume was $105 \%$ of volume in September 2019

breast imaging workflow. Patient intake questionnaires were modified to include questions regarding vaccination status, date, and site of administration. Some patients were offended by the questions and had to be reassured by staff that the questions were medically necessary for their breast imaging care, to obviate unnecessary biopsies and screening callbacks. Screening patients were encouraged to schedule screening exams either prior to initial dose of COVID-19 vaccination or 4-6 weeks following the last dose of COVID-19 vaccination. 


\section{Conclusion}

Since COVID-19 emerged as a full-fledged pandemic in early 2020, there have been several guidelines issued regarding management of breast care during the pandemic, including statements from the Society of Breast Imaging and a comprehensive joint statement from the American Society of Breast Surgeons, The National Accreditation Program for Breast Centers, the National Comprehensive Care Network, the Commission on Cancer, and the American College of Radiology [7, 8•]. A review of the existing published guidelines is beyond the scope of this manuscript. Instead, by sharing our experience, we hope to provide others with insights into the practical solutions for the implementation of pandemic-related modified protocols for breast imaging services.

Funding Not applicable.

Data Availability Not applicable.

Code Availability Not applicable.

\section{Declarations}

Conflict of interest Not applicable.

Ethical Approval Not applicable.

Consent to Participate Not applicable.

Consent for Publication Not applicable.

\section{References}

Papers of particular interest, published recently, have been highlighted as:
- Of importance

1. Goldstein J, McKinley J. Coronavirus in NY: Manhattan Woman is first confirmed case in state. The New York Times. 2020. https:// www.nytimes.com/2020/03/01/nyregion/new-york-coronvirus-con firmed.html.

2. Gold M, Ferre-Sadurni L. 9 New cases of coronavirus in NY. All connected to Westchester Man. The New York Times. 2020. https://www.nytimes.com/2020/03/04/nyregion/coronavirus-nycyeshiva-university.html.

3. McKinely J, Sandoval E. Coronavirus in NY: Cuomo declares state of emergency. The New York Times. 2020. https://www. nytimes.com/2020/03/07/nyregion/coronavirus-new-york-queens. html.

4. Evelyn K. Here's What a 'stay home' order means for New York. The Guardian. 2020. https://www.theguardian.com/us-news/2020/ mar/20/new-york-90-day-stay-home-order-what-it-means.

5. McKinley J. Here's Cuomo's Plan for Reopening New York. The New York Times. 2020.https://www.nytimes.com/2020/05/04/ nyregion/coronavirus-reopen-cuomo-ny.html.

6. Rapier G. New York City is relaxing its coronavirus restrictions after 78 days. These are the businesses that can reopen. Business Insider. 2020. https://www.businessinsider.com/new-york-cityphase-1-reopening-rules-explained-businesses-workers-2020-6.

7. Society of Breast Imaging Statement on Breast Imaging during the COVID-19 Pandemic. 2020. https://www.sbi-online.org/Portals/0/ Position\%20Statements/2020/society-of-breast-imaging-statementon-breast-imaging-during-COVID19-pandemic.pdf. Accessed 22 June 2021.

8. Recommendations for Prioritization, Treatment and Triage of Breast Cancer Patients During the COVID-19 Pandemic. The COVID-19 Pandemic Consortium: Representatives from the American Society of Breast surgeons (AsBrS), the National Accreditation Program for breast Centers (NABPC), the National Comprehensive Care Network ( $\mathrm{NCCN})$, the Commission on Cancer (CoC), and American College of Radiology (ACR). https://www.facs.org/-/media/files/quality-programs/napbc/asbrs_ napbc_coc_nccn_acr_bc_covid_consortium_recommendations.ashx. Accessed 22 June 2021. Joint statement from multiple breast care subspecialties providing guidelines for prioritizing care in the setting of the COVID-19 pandemic.

Publisher's Note Springer Nature remains neutral with regard to jurisdictional claims in published maps and institutional affiliations. 